\title{
Abnormal Expression of DNA Double-Strand Breaks Related Genes, ATM and GammaH2AX, in Thyroid Carcinoma
}

\author{
Jin-lin Hu, ${ }^{1}$ Si-si Hu, ${ }^{2}$ Xiu-xiu Hou, ${ }^{3}$ Xin Zhu, ${ }^{3}$ Jun Cao, ${ }^{3}$ Lie-hao Jiang, ${ }^{3}$ and Ming-hua Ge ${ }^{4}$ \\ ${ }^{1}$ Department of Pathology, Zhejiang Province Cancer Hospital, Hangzhou 310022, China \\ ${ }^{2}$ Emergency Department, The First Affiliated Hospital of Wenzhou Medical University, Wenzhou 325000, China \\ ${ }^{3}$ Zhejiang Cancer Research Institute, Zhejiang Province Cancer Hospital, Hangzhou 310022, China \\ ${ }^{4}$ Department of Head and Neck Surgery, Zhejiang Province Cancer Hospital, Hangzhou 310022, China
}

Correspondence should be addressed to Ming-hua Ge; gemh@zjcc.org.cn

Received 16 December 2014; Revised 11 February 2015; Accepted 5 March 2015

Academic Editor: Sabrina Corbetta

Copyright (C) 2015 Jin-lin Hu et al. This is an open access article distributed under the Creative Commons Attribution License, which permits unrestricted use, distribution, and reproduction in any medium, provided the original work is properly cited.

\begin{abstract}
ATM and $\gamma \mathrm{H} 2 \mathrm{AX}$ play a vital role in the detection of DNA double-strand breaks (DSB) and DNA damage response (DDR). This study aims to investigate ATM and $\gamma \mathrm{H} 2 \mathrm{AX}$ expression in thyroid cancer and discuss possible relationship between thyroid function tests and DNA damage. The expression of ATM and $\gamma \mathrm{H} 2 \mathrm{AX}$ was detected by immunohistochemistry in 30 cases of benign nodular goiter, 110 cases of well differentiated thyroid cancer, 22 cases of poorly differentiated thyroid cancer, and 21 cases of anaplastic thyroid cancer. Clinicopathological features, including differentiation stages, distant metastasis, lymph node metastasis, T classification, TNM stage, and tests of thyroid functions (TPOAb, Tg Ab, T3, FT3, T4, FT4, TSH, and Tg), were reviewed and their associations with $\gamma \mathrm{H} 2 \mathrm{AX}$ and ATM were analyzed. $\gamma \mathrm{H} 2 \mathrm{AX}$ and ATM expressed higher in thyroid cancer tissues than in benign nodular goiter and normal adjacent tissues. $\gamma \mathrm{H} 2 \mathrm{AX}$ was correlated with ATM in thyroid cancer. Both $\gamma \mathrm{H} 2 \mathrm{AX}$ and ATM expression were associated with FT3. $\gamma \mathrm{H} 2 \mathrm{AX}$ was also associated with T classification, TNM stage, FT4, TSH, and differentiation status. Therefore both of ATM and $\gamma \mathrm{H} 2 \mathrm{AX}$ seem to correlate with thyroid hormones and $\gamma \mathrm{H} 2 \mathrm{AX}$ plays a role in the differentiation status of thyroid cancer.
\end{abstract}

\section{Introduction}

Thyroid cancer, which is related to radiation exposure, represents the most common malignancy in the endocrine system. A marked increase in the incidence of thyroid cancer has occurred in recent years, in part due to the improvement in the diagnostic approach $[1,2]$. Among them, anaplastic thyroid carcinoma is the most aggressive form and remains a medical challenge, with a poor survival time from diagnosis [3]. However, the genetic predisposition to thyroid cancer has still not been clearly elucidated.

DNA double-strand break (DSB), the primary lesions induced by radiation exposure, might be involved in thyroid carcinoma development. A single misrepaired DSB could be sufficient to lead to loss of large DNA fragments, abnormal mitosis, and even activation of cell death process $[4,5]$. To reverse and diminish severe DNA damage, cells develop different mechanisms, including DNA recombination and repair, cell cycle arrest, and apoptosis [4]. Both ATM and $\mathrm{H} 2 \mathrm{AX}$ have been proved to be implicated in maintaining genomic stability and response to DSB [6-8].

ATM gene deficiency leads to the syndrome named ataxia telangiectasia and the latter is characterized by genome instability, radiosensitivity, progressive ataxia, and susceptibility to malignancy [9]. After being recruited to the DSB sites, autoactivated ATM phosphorylates various substrates, including $\mathrm{H} 2 \mathrm{AX}, \mathrm{NBS1}, \mathrm{CHK} 2$, and $\mathrm{p} 53$, thereby mediating DSB repairing $[4,8]$. Some specific ATM alleles were correlated with the increased risk of various cancers, including lung, breast, and prostate cancer [10-12]. However, it has been proved that some specific ATM alleles could be protective in cutaneous melanoma [13]. Different studies demonstrated different roles of ATM in thyroid cancer. A research conducted by Wójcicka et al. revealed that the ATM variant ATM rs1801516 was not associated with PTC risk but could modify BRCA1 related risk [14]. Kang et al. also found no 
positive associations between ATM polymorphisms and PTC risk [15]. Some other researches demonstrated that ATM polymorphisms might be involved in the development of thyroid cancer $[9,16-18]$. Accumulation of H2AX phosphorylation $(\gamma \mathrm{H} 2 \mathrm{AX})$, which could be mediated by ATM, has been detected in various cancers [19-22]. Activation of ATM and accumulation of $\gamma \mathrm{H} 2 \mathrm{AX}$ could be a cellular response to DNA damage [6-8]. In the previous study, we have found that $\gamma \mathrm{H} 2 \mathrm{AX}$ expression was increased in papillary thyroid cancer (PTC) patients and was reversely related to lymph node metastasis and TNM stage [23]. Recent researches demonstrated that $\gamma \mathrm{H} 2 \mathrm{AX}$ was increased in human primary thyrocytes disposed by ionizing radiation and in differentiated thyroid cancer patients undergoing ${ }^{131}$ I therapy [24-26]. However, the role of ATM and $\gamma \mathrm{H} 2 \mathrm{AX}$ in thyroid cancer has not been completely elucidated.

Various hormones, including estrogen, could induce DNA damage, thus contributing to carcinogenesis [27]. Recent studies found various thyroid disorders in thyroid cancer patients and found that abnormal FT3, FT4, and TSH levels were related to thyroid cancer risk [28]. The thyroid hormones exert momentous actions on energy metabolism. The increase in oxygen consumption led by thyroid hormones induces the generation of reactive oxygen species and reactive nitrogen species. And, in response, more cellular antioxidants could be consumed and antioxidant enzymes could be inactivated, thereby promoting oxidative stress and oxidative DNA damage. Thus, we hypothesize that there is a relation between thyroid disorders and DNA damage. However, there is lack of data about the relationship between thyroid functions and DSB-related genes.

To investigate the expression of $\gamma \mathrm{H} 2 \mathrm{AX}$ and ATM in thyroid cancer, 30 cases of benign nodular goiter, 110 cases of well differentiated thyroid cancer, 22 cases of poorly differentiated thyroid cancer, and 21 cases of anaplastic thyroid cancer were included in this study. To determine if thyroid hormones play a role in DNA damage, data of thyroid function tests was collected and analysis was made to discuss the relationship between $\gamma \mathrm{H} 2 \mathrm{AX}$ and ATM expression and thyroid function tests in thyroid cancer in this study.

\section{Materials and Methods}

2.1. Tissue Specimens. 30 patients diagnosed with benign nodular goiter and 153 patients diagnosed with thyroid cancer were included in this study. Among the thyroid cancer group, 80 cases were diagnosed coexisting with benign nodular goiter. The thyroid cancer patients were identified between January 2001 and August 2014, while the benign nodular goiter patients were identified between January 2012 and June 2012 in Zhejiang Province Cancer Hospital, China. A final diagnosis of thyroid cancer and benign nodular goiter was confirmed by at least two experienced pathologists. Various clinicopathological features, including age, gender, differentiation stages, lymph node metastasis, and distant metastasis, were captured according to the medical records. $\mathrm{T}$ classification and TNM stage of the cancer patients were evaluated based on the 2002 edition of American Joint
Committee on Cancer (AJCC) TNM staging criteria. Tests of thyroid functions, including peroxidase antibody (TPOAb), thyroglobulin antibody (TgAb), triiodothyronine (T3), basal plasma free T3 (FT3), thyroxine (T4), basal plasma free T4 (FT4), thyroid-stimulating hormone (TSH), and thyroglobulin (Tg) levels, were also studied prior to surgery and they were evaluated according to the following standards (Table 1). 30 patients diagnosed with benign nodular goiter, including 29 females and 1 male, ranged from 36 to 70 years old (49.0 \pm 8.0). 153 patients diagnosed with thyroid cancer, including 104 females and 49 males, ranged from 18 to 78 years old ( $48.8 \pm$ 13.9). Among the cancer patients, lymph node metastasis was found in 107 (69.9\%) cases, while distant metastasis was detected in $19(12.4 \%)$ cases. According to the postoperative histopathological examination, 110 (52.3\%) cases were well differentiated, 22 (14.4\%) were poorly differentiated, and the other $21(13.7 \%)$ were anaplastic. None of all the thyroid cancer patients had ever received anticancer treatment before surgery.

\subsection{Tissue Microarray Construction and Immunohistochem-} istry. By reviewing HE stained slides, paraffin blocks containing representative area from each specimens were chosen and extracted with the TM-1 tissue microarray kit (Changzhou Ruipin Precision Instrument Co., Ltd., China). The cores were subsequently placed and embedded in blank paraffin blocks. The blocks were incubated at $60^{\circ} \mathrm{C}$ for 30 minutes and cooled at room temperature for better fixation of the tissue.

$3 \mu \mathrm{m}$ thick sections, obtained from tissue microarrays blocks or tissue paraffin blocks, were used for immunohistochemistry. The sections were then placed in xylene for deparaffinization and sequentially several graded alcohol dilutions for hydration. To detect $\gamma \mathrm{H} 2 \mathrm{AX}$ expression, sections were boiled in a pressure kettle for 1.5 minutes in citrate buffer ( $\mathrm{pH}$ 6.0) and cooled to room temperature for antigen retrieval. After endogenous peroxidase activity was blocked, the slices were incubated with anti- $\gamma \mathrm{H} 2 \mathrm{AX}$ antibody (pS139, Cat:\#2212-1, EPITOMICS, CA, USA) (1:200 dilution) for 90 minutes and subsequently the secondary antibody (DAKO, Denmark) for 30 minutes.

To detect ATM expression, sections were boiled in a microwave oven for 15 minutes in EDTA buffer $(\mathrm{pH} 9.0$ ) for antigen retrieval and cooled to room temperature. After endogenous peroxidase activity was blocked, the slices were incubated with anti-ATM antibody (ab32420, Abcam) (1:200 dilution) for 90 minutes and subsequently the secondary antibody (DAKO, Denmark) for 30 minutes.

All slices were then treated with DAB for 5 minutes and subsequently counterstained with hematoxylin. All the sections were reviewed by at least two experienced pathologists. The positive cell percentages $0 \%, 0-25 \%, 26 \%-50 \%, 51-75 \%$, and $>75 \%$ were defined as $0,1,2,3$, and 4 , respectively. The positive staining intensity were defined as $0,1,2$, and 3 for no staining, light yellow, yellow brown and brown, respectively. Semiquantitative expression levels of ATM and $\gamma \mathrm{H} 2 \mathrm{AX}$ were evaluated by multiplying the distribution and intensity score. Those with a final score $<5$ were defined as low expression and those $\geqq 5$ were considered high expression. 
2.3. Statistical Analysis. All tests were two-sided and carried out with SPSS version 18.0. $\gamma \mathrm{H} 2 \mathrm{AX}$ and ATM expression were compared between cancer tissues and benign tissues using $\chi^{2}$ test. The correlation between $\gamma \mathrm{H} 2 \mathrm{AX}$ and ATM was also evaluated by $\chi^{2}$ test. In the cancer group, the associations between the expression of $\gamma \mathrm{H} 2 \mathrm{AX}$ and ATM and clinicopathological features were carried out by $\chi^{2}$ test. $P \leqslant$ 0.05 was defined as statistically significant.

\section{Results}

3.1. Expression of $\gamma H 2 A X$ and ATM in Thyroid Cancer and in Benign Tissues. $\gamma \mathrm{H} 2 \mathrm{AX}$ was mainly expressed in nucleus (Figure 1), while ATM was mainly expressed in both nucleus and cytoplasm in thyroid tissues (Figure 2).

In benign nodular goiter patients, $\gamma \mathrm{H} 2 \mathrm{AX}$ was highly expressed in 6 of $30(20.0 \%)$ benign nodular goiter tissues and 3 of $30(10.0 \%)$ normal adjacent tissues, with no statistical significance $(P=0.472)$, while ATM was highly expressed in 3 of $30(10.0 \%)$ benign nodular goiter tissues and 2 of 30 $(6.7 \%)$ normal adjacent tissues, with no statistical significance $(P=1.000)$.

In thyroid cancer, high $\gamma \mathrm{H} 2 \mathrm{AX}$ expression was detected in 109 of $153(71.2 \%)$ cancer tissues and 9 of 153 (5.9\%) normal adjacent tissues, with statistical significance $(P<0.001)$, while high ATM expression was detected in 53 of 153 (34.6\%) cancer tissues and 9 of $153(5.9 \%)$ normal adjacent tissues, with statistical significance $(P<0.001)$.

Among 153 thyroid cancer patients, 80 cases were coexisting with benign nodular goiter. In this coexisting group, 76 of $80(95.0 \%)$ cancer tissues and 10 of $80(12.5 \%)$ benign nodular goiter tissues showed high expression of $\gamma \mathrm{H} 2 \mathrm{AX}(P<0.001)$. 31 of $80(38.8 \%)$ cancer tissues and 3 of $80(3.8 \%)$ benign nodular goiter tissues showed high expression of ATM $(P<$ 0.001).

Overall, both $\gamma \mathrm{H} 2 \mathrm{AX}$ and ATM expressed higher in thyroid cancer tissues than in benign nodular goiter and normal adjacent tissues, while benign nodular goiter and normal adjacent tissues showed no significant difference in $\gamma \mathrm{H} 2 \mathrm{AX}$ expression. They showed no significant difference in ATM expression as well.

In thyroid cancer group, both ATM and $\gamma \mathrm{H} 2 \mathrm{AX}$ were highly expressed in 45 cases and lowly expressed in 36 cases. Analysis between ATM and $\gamma \mathrm{H} 2 \mathrm{AX}$ expression showed statistical significance $(P=0.007)$ (Table 2$)$. ATM expression was correlated with $\gamma \mathrm{H} 2 \mathrm{AX}$ expression in thyroid cancer in this study.

3.2. Clinical Significance of $\gamma H 2 A X$ and ATM in Thyroid Cancer. Association between $\gamma \mathrm{H} 2 \mathrm{AX}$ expression and various clinicopathological features of thyroid cancer was analyzed (Table 3). $\gamma \mathrm{H} 2 \mathrm{AX}$ expression was related to $\mathrm{T}$ classification, and the level was higher in earlier $\mathrm{T}$ stage than that in later $\mathrm{T}$ stage $(<0.001), \gamma \mathrm{H} 2 \mathrm{AX}$ expression was also correlated with TNM stage $(<0.001)$ and differentiation status $(<0.001)$. Well differentiated thyroid cancer tissues showed higher $\gamma \mathrm{H} 2 \mathrm{AX}$ expression level than poorly differentiated thyroid cancer tissues, while the latter showed higher $\gamma \mathrm{H} 2 \mathrm{AX}$ expression level
TABLE 1: The evaluation standards of the tests of thyroid functions.

\begin{tabular}{cccccc}
\hline $\begin{array}{c}\text { Thyroid } \\
\text { hormone }\end{array}$ & \multicolumn{2}{c}{ Level } & $\begin{array}{l}\text { Thyroid } \\
\text { hormone }\end{array}$ \\
\hline TgAb & & & TPOAb & \\
$\mathrm{N}$ & $0-115$ & $\mathrm{U} / \mathrm{mL}$ & $\mathrm{N}$ & $0-34$ & $\mathrm{U} / \mathrm{mL}$ \\
$\mathrm{H}$ & $>115$ & $\mathrm{U} / \mathrm{mL}$ & $\mathrm{H}$ & $>34$ & $\mathrm{U} / \mathrm{mL}$ \\
$\mathrm{T} 3$ & & & $\mathrm{~T} 4$ & & \\
$\mathrm{~L}$ & $<0.6$ & $\mathrm{ng} / \mathrm{mL}$ & $\mathrm{L}$ & $<4.5$ & $\mu \mathrm{g} / \mathrm{dL}$ \\
$\mathrm{N}$ & $0.6-1.81$ & $\mathrm{ng} / \mathrm{mL}$ & $\mathrm{N}$ & $4.5-10.9$ & $\mu \mathrm{g} / \mathrm{dL}$ \\
$\mathrm{H}$ & $>1.81$ & $\mathrm{ng} / \mathrm{mL}$ & $\mathrm{H}$ & $>10.9$ & $\mu \mathrm{g} / \mathrm{dL}$ \\
$\mathrm{FT3}$ & & & $\mathrm{FT} 4$ & & \\
$\mathrm{~L}$ & $<2.3$ & $\mathrm{pg} / \mathrm{mL}$ & $\mathrm{L}$ & $<0.89$ & $\mathrm{ng} / \mathrm{dL}$ \\
$\mathrm{N}$ & $2.3-4.2$ & $\mathrm{pg} / \mathrm{mL}$ & $\mathrm{N}$ & $0.89-1.76$ & $\mathrm{ng} / \mathrm{dL}$ \\
$\mathrm{H}$ & $>4.2$ & $\mathrm{pg} / \mathrm{mL}$ & $\mathrm{H}$ & $>1.76$ & $\mathrm{ng} / \mathrm{dL}$ \\
$\mathrm{TSH}$ & & & $\mathrm{Tg}$ & & \\
$\mathrm{L}$ & $<0.35$ & $\mu \mathrm{IU} / \mathrm{mL}$ & $\mathrm{L}$ & $<1.4$ & $\mathrm{ng} / \mathrm{mL}$ \\
$\mathrm{N}$ & $0.35-5.5$ & $\mu \mathrm{IU} / \mathrm{mL}$ & $\mathrm{N}$ & $1.4-78$ & $\mathrm{ng} / \mathrm{mL}$ \\
$\mathrm{H}$ & $>5.5$ & $\mu \mathrm{IU} / \mathrm{mL}$ & $\mathrm{H}$ & $>78$ & $\mathrm{ng} / \mathrm{mL}$ \\
\hline
\end{tabular}

L represents low expression level; $\mathrm{N}$ represents normal expression level; $\mathrm{H}$ represents high expression level.

TABLE 2: Correlation between ATM and $\gamma \mathrm{H} 2 \mathrm{AX}$ in thyroid cancer.

\begin{tabular}{|c|c|c|c|c|c|c|}
\hline & \multirow{2}{*}{ Number } & \multicolumn{2}{|c|}{ ATM-low } & \multicolumn{2}{|c|}{ ATM-high } & \multirow{2}{*}{$P$ value } \\
\hline & & $N$ & $\%$ & $N$ & $\%$ & \\
\hline$\gamma \mathrm{H} 2 \mathrm{AX}-\mathrm{low}$ & 44 & 36 & $81.8 \%$ & 8 & $22.2 \%$ & 0.007 \\
\hline$\gamma \mathrm{H} 2 \mathrm{AX}$-high & 109 & 64 & $58.7 \%$ & 45 & $41.3 \%$ & \\
\hline
\end{tabular}

than anaplastic thyroid cancer tissues. $\gamma \mathrm{H} 2 \mathrm{AX}$ expression was also associated with several tests of thyroid functions including FT3 $(P=0.043)$, FT4 $(P=0.009)$, and TSH $(<0.001)$. Other clinicopathological features showed no correlation with $\gamma \mathrm{H} 2 \mathrm{AX}$ expression level, including gender, distant metastasis, lymph node metastasis, TPOAb, TgAb, T3, $\mathrm{T} 4$, and $\mathrm{Tg}$.

Association between ATM expression and clinicopathological features of thyroid cancer was also analyzed (Table 4). Different pathologic type of thyroid cancer tissues showed different expression level of ATM, with statistically significant differences $(P=0.05)$. Anaplastic thyroid cancer tissues showed higher ATM expression than well differentiated thyroid cancer tissues, while the latter showed higher ATM expression than poorly differentiated thyroid cancer tissues. However no distinct correlation has been found between ATM expression and differentiation of thyroid cancer. ATM expression level was also associated with several tests of thyroid functions, including FT3 $(P=0.009)$ and T4 $(P=0.037)$. ATM expression level was not correlated with other clinicopathological features, including distant metastasis, $\mathrm{T}$ statues, lymph node metastasis, TNM stage, TPOAb, TgAb, T3, FT4, TSH, and $\mathrm{Tg}$. 


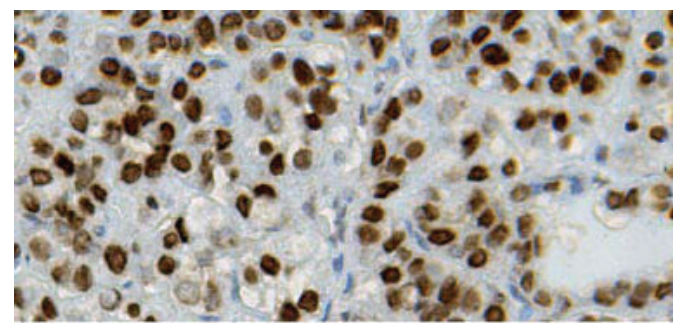

(A)

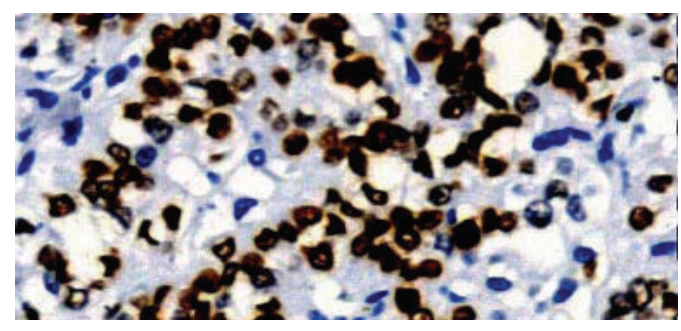

(B)

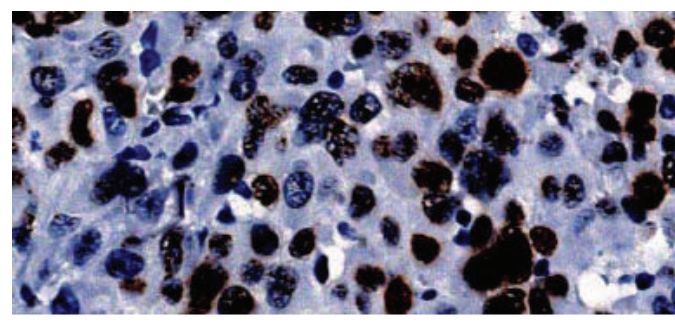

(C)

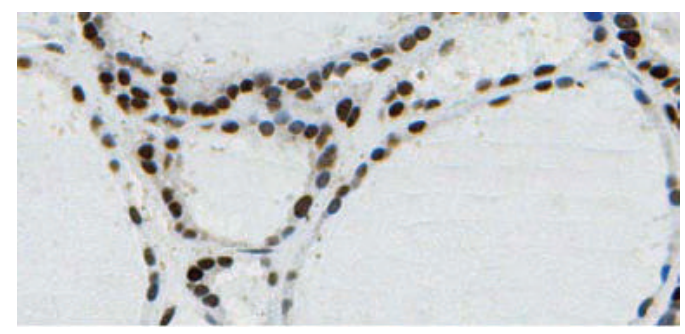

(D)

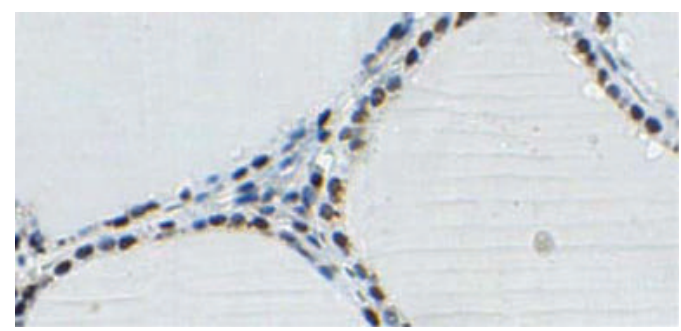

(E)

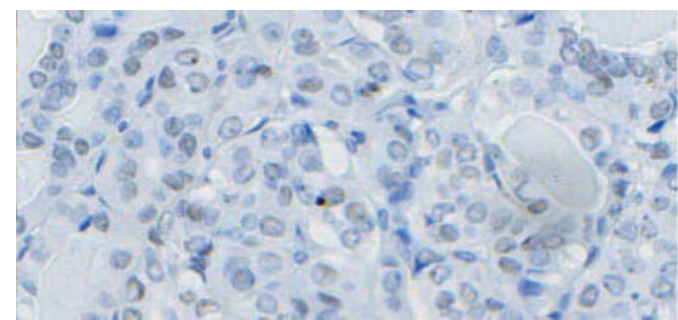

(a)

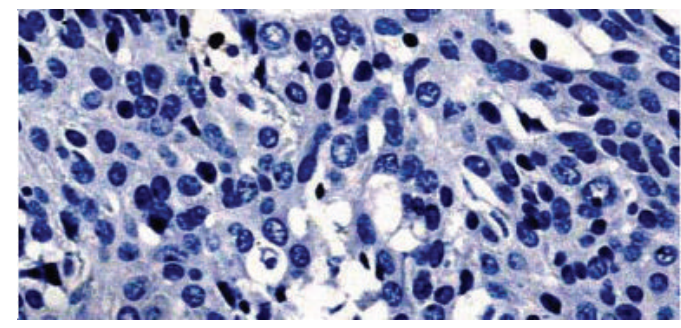

(b)

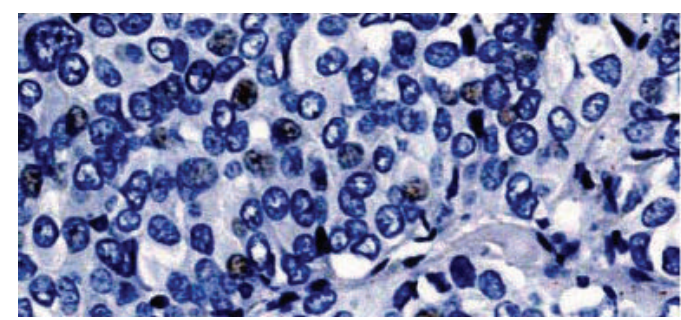

(c)

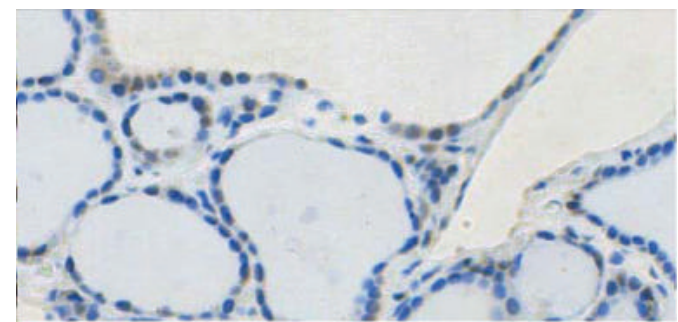

(d)

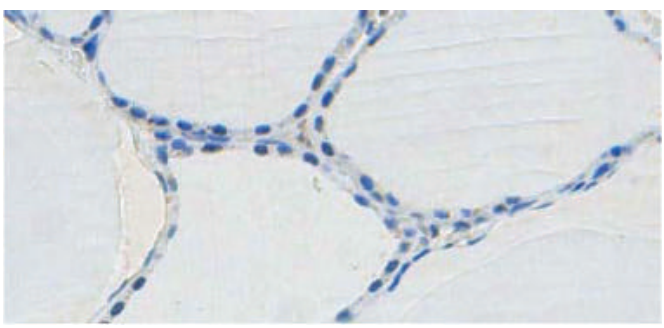

(e)

FIGURE 1: $\gamma \mathrm{H} 2 \mathrm{AX}$ expression in thyroid tissues. (A) High expression of $\gamma \mathrm{H} 2 \mathrm{AX}$ in well differentiated thyroid cancer. (a) Low expression of $\gamma \mathrm{H} 2 \mathrm{AX}$ in well differentiated thyroid cancer. (B) High expression of $\gamma \mathrm{H} 2 \mathrm{AX}$ in poorly differentiated thyroid cancer. (b) Low expression of $\gamma \mathrm{H} 2 \mathrm{AX}$ in poorly differentiated thyroid cancer. (C) High expression of $\gamma \mathrm{H} 2 \mathrm{AX}$ in anaplastic thyroid cancer. (c) Low expression of $\gamma \mathrm{H} 2 \mathrm{AX}$ in anaplastic thyroid cancer. (D) High expression of $\gamma \mathrm{H} 2 \mathrm{AX}$ in benign nodular goiter tissues. (d) Low expression of $\gamma \mathrm{H} 2 \mathrm{AX}$ in benign nodular goiter tissues. (E) High expression of $\gamma \mathrm{H} 2 \mathrm{AX}$ in normal adjacent tissues. (e) Low expression of $\gamma \mathrm{H} 2 \mathrm{AX}$ in normal adjacent tissues. Original magnification of all images, $\times 200$. 


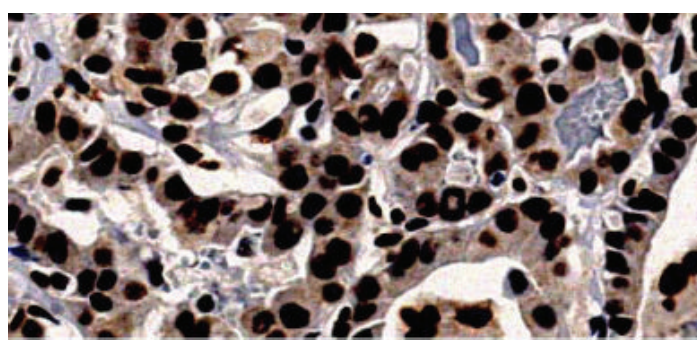

(A)

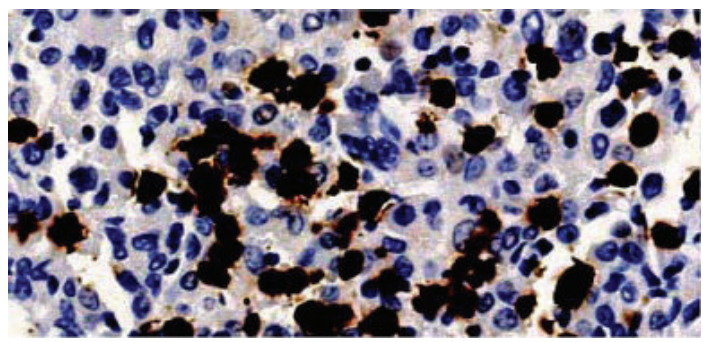

(B)

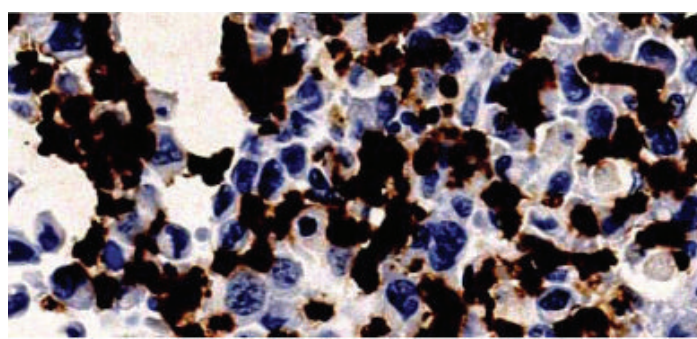

(C)

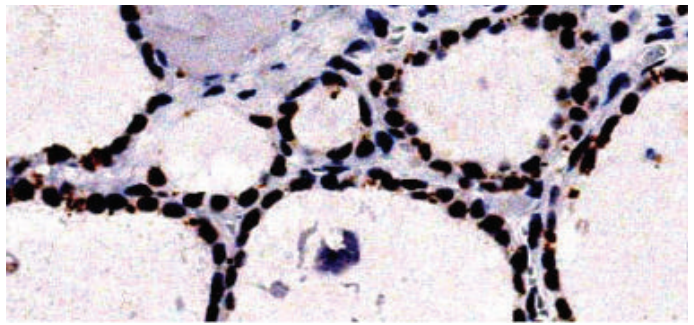

(D)

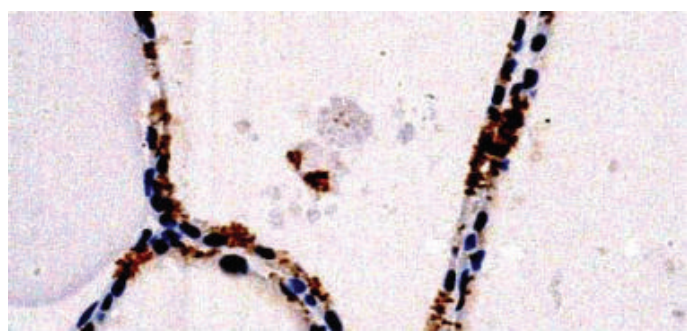

(E)

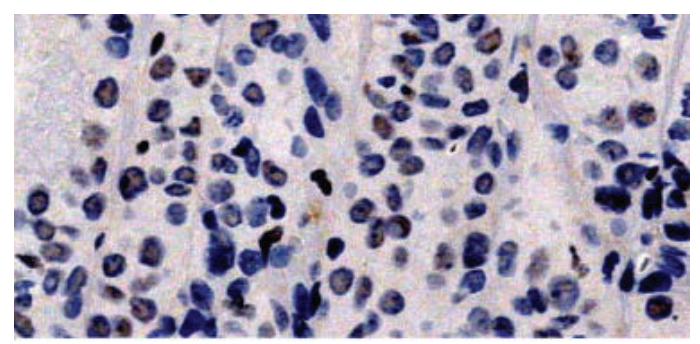

(a)

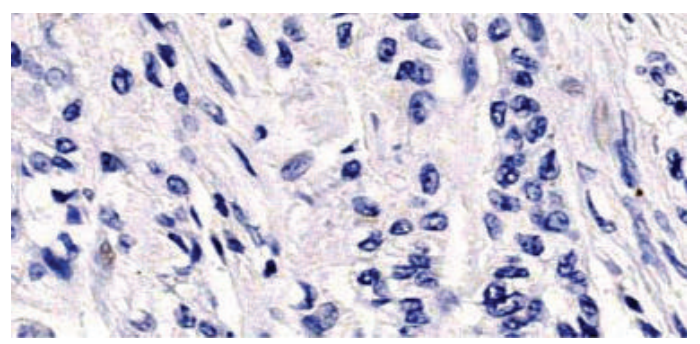

(b)

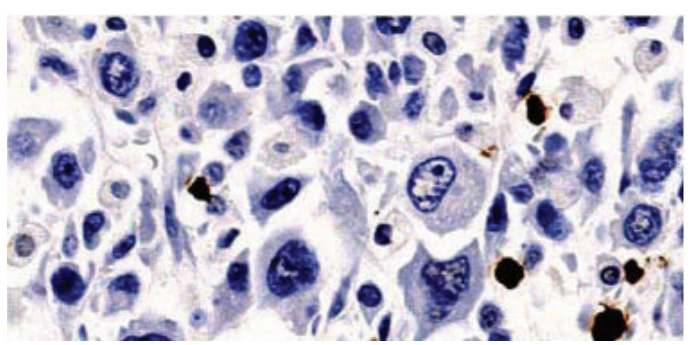

(c)

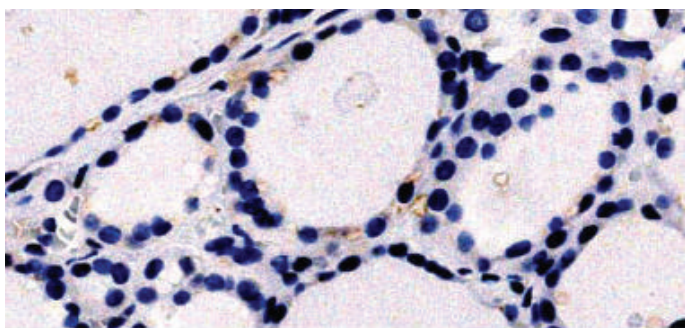

(d)

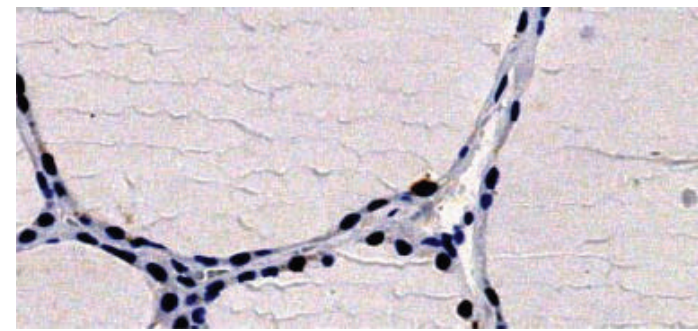

(e)

FIgURE 2: ATM expression in thyroid tissues. (A) High expression of ATM in well differentiated thyroid cancer. (a) Low expression of ATM in well differentiated thyroid cancer. (B) High expression of ATM in poorly differentiated thyroid cancer. (b) Low expression of ATM in poorly differentiated thyroid cancer. (C) High expression of ATM in anaplastic thyroid cancer. (c) Low expression of ATM in anaplastic thyroid cancer. (D) High expression of ATM in benign nodular goiter tissues. (d) Low expression of ATM in benign nodular goiter tissues. (E) High expression of ATM in normal adjacent tissues. (e) Low expression of ATM in normal adjacent tissues. Original magnification of all images, $\times 200$. 
TABLE 3: Association between $\gamma \mathrm{H} 2 \mathrm{AX}$ expression and clinicopathological features.

\begin{tabular}{|c|c|c|c|c|c|c|}
\hline \multirow{3}{*}{ Total number } & \multirow{2}{*}{ Number } & \multicolumn{2}{|c|}{$\gamma \mathrm{H} 2 \mathrm{AX}-\mathrm{low}$} & \multicolumn{2}{|c|}{$\gamma \mathrm{H} 2 \mathrm{AX}$-high } & \multirow{2}{*}{$P$ value } \\
\hline & & $N$ & $\%$ & $N$ & $\%$ & \\
\hline & \multicolumn{6}{|c|}{ Total number } \\
\hline Distant metastasis & & & & & & 0.17 \\
\hline- & 134 & 36 & $26.9 \%$ & 98 & $73.1 \%$ & \\
\hline+ & 19 & 8 & $42.1 \%$ & 11 & $57.9 \%$ & \\
\hline $\mathrm{T}$ classification & & & & & & $<0.001$ \\
\hline 1 & 11 & 0 & $0.0 \%$ & 11 & $100.0 \%$ & \\
\hline 2 & 7 & 1 & $14.3 \%$ & 6 & $85.7 \%$ & \\
\hline 3 & 84 & 13 & $15.5 \%$ & 71 & $84.5 \%$ & \\
\hline 4 & 51 & 30 & $58.8 \%$ & 21 & $41.2 \%$ & \\
\hline Lymph node metastasis & & & & & & 0.1 \\
\hline- & 46 & 9 & $19.6 \%$ & 37 & $80.4 \%$ & \\
\hline+ & 107 & 35 & $32.7 \%$ & 72 & $67.3 \%$ & \\
\hline TNM stage (2002 AJCC) & & & & & & $<0.001$ \\
\hline I & 49 & 5 & $10.2 \%$ & 44 & $89.8 \%$ & \\
\hline II & 7 & 3 & $42.9 \%$ & 4 & $57.1 \%$ & \\
\hline III & 42 & 5 & $11.9 \%$ & 37 & $88.1 \%$ & \\
\hline IV & 55 & 31 & $56.4 \%$ & 24 & $43.6 \%$ & \\
\hline Differentiation & & & & & & $<0.001$ \\
\hline Well differentiated & 110 & 11 & $10.0 \%$ & 99 & $90.0 \%$ & \\
\hline Poorly differentiated & 22 & 15 & $68.2 \%$ & 7 & $31.8 \%$ & \\
\hline Anaplastic & 21 & 18 & $85.7 \%$ & 3 & $14.3 \%$ & \\
\hline TPOAb & & & & & & 0.376 \\
\hline $\mathrm{N}$ & 120 & 30 & $25.0 \%$ & 90 & $75.0 \%$ & \\
\hline $\mathrm{H}$ & 27 & 9 & $33.3 \%$ & 18 & $66.7 \%$ & \\
\hline \multicolumn{7}{|l|}{$\operatorname{TgAb}$} \\
\hline $\mathrm{N}$ & 119 & 30 & $25.2 \%$ & 89 & $74.8 \%$ & 0.455 \\
\hline $\mathrm{H}$ & 28 & 9 & $32.1 \%$ & 19 & $67.9 \%$ & \\
\hline \multicolumn{7}{|l|}{$\mathrm{T} 3$} \\
\hline $\mathrm{L}$ & 6 & 1 & $16.7 \%$ & 5 & $83.3 \%$ & 0.421 \\
\hline $\mathrm{N}$ & 141 & 40 & $28.4 \%$ & 101 & $71.6 \%$ & \\
\hline $\mathrm{H}$ & 2 & 0 & $0.0 \%$ & 2 & $100.0 \%$ & \\
\hline \multicolumn{7}{|l|}{ FT3 } \\
\hline $\mathrm{L}$ & 10 & 5 & $50.0 \%$ & 5 & $50.0 \%$ & 0.043 \\
\hline $\mathrm{N}$ & 130 & 31 & $23.8 \%$ & 99 & $76.2 \%$ & \\
\hline $\mathrm{H}$ & 9 & 5 & $55.6 \%$ & 4 & $44.4 \%$ & \\
\hline $\mathrm{T} 4$ & & & & & & 0.308 \\
\hline $\mathrm{L}$ & 3 & 1 & $33.3 \%$ & 2 & $66.7 \%$ & \\
\hline $\mathrm{N}$ & 133 & 34 & $25.6 \%$ & 99 & $74.4 \%$ & \\
\hline $\mathrm{H}$ & 13 & 6 & $46.2 \%$ & 7 & $53.8 \%$ & \\
\hline FT4 & & & & & & 0.009 \\
\hline $\mathrm{L}$ & 7 & 4 & $57.1 \%$ & 3 & $42.9 \%$ & \\
\hline $\mathrm{N}$ & 135 & 32 & $23.7 \%$ & 103 & $76.3 \%$ & \\
\hline $\mathrm{H}$ & 7 & 5 & $71.4 \%$ & 2 & $28.6 \%$ & \\
\hline TSH & & & & & & $<0.001$ \\
\hline $\mathrm{L}$ & 7 & 6 & $85.7 \%$ & 1 & $14.3 \%$ & \\
\hline $\mathrm{N}$ & 133 & 27 & $20.3 \%$ & 106 & $79.7 \%$ & \\
\hline $\mathrm{H}$ & 9 & 8 & $88.9 \%$ & 1 & $11.1 \%$ & \\
\hline $\mathrm{Tg}$ & & & & & & 0.082 \\
\hline $\mathrm{L}$ & 14 & 5 & $35.7 \%$ & 9 & $64.3 \%$ & \\
\hline $\mathrm{N}$ & 97 & 20 & $20.6 \%$ & 77 & $79.4 \%$ & \\
\hline $\mathrm{H}$ & 36 & 14 & $38.9 \%$ & 22 & $61.1 \%$ & \\
\hline
\end{tabular}


TABLE 4: Association between ATM expression and clinicopathological features.

\begin{tabular}{|c|c|c|c|c|c|c|}
\hline & \multirow{2}{*}{ Number } & \multicolumn{2}{|c|}{$\gamma \mathrm{H} 2 \mathrm{AX}-\mathrm{low}$} & \multicolumn{2}{|c|}{$\gamma \mathrm{H} 2 \mathrm{AX}$-high } & \multirow{2}{*}{$P$ value } \\
\hline & & $N$ & $\%$ & $N$ & $\%$ & \\
\hline \multicolumn{7}{|l|}{ Total number } \\
\hline Distant metastasis & & & & & & 0.183 \\
\hline- & 134 & 85 & $63.4 \%$ & 49 & $36.6 \%$ & \\
\hline+ & 19 & 15 & $78.9 \%$ & 4 & $21.1 \%$ & \\
\hline \multicolumn{7}{|l|}{$\mathrm{T}$ classification } \\
\hline 1 & 11 & 9 & $81.8 \%$ & 2 & $18.2 \%$ & 0.287 \\
\hline 2 & 7 & 6 & $85.7 \%$ & 1 & $14.3 \%$ & \\
\hline 3 & 84 & 51 & $60.7 \%$ & 33 & $39.3 \%$ & \\
\hline 4 & 51 & 34 & $66.7 \%$ & 17 & $33.3 \%$ & \\
\hline Lymph node metastasis & & & & & & 0.132 \\
\hline- & 46 & 26 & $56.5 \%$ & 20 & $43.5 \%$ & \\
\hline+ & 107 & 74 & $69.2 \%$ & 33 & $30.8 \%$ & \\
\hline TNM stage (2002 AJCC) & & & & & & 0.602 \\
\hline I & 49 & 33 & $67.3 \%$ & 16 & $32.7 \%$ & \\
\hline II & 7 & 6 & $85.7 \%$ & 1 & $14.3 \%$ & \\
\hline III & 42 & 26 & $61.9 \%$ & 16 & $38.1 \%$ & \\
\hline IV & 55 & 35 & $63.6 \%$ & 20 & $36.4 \%$ & \\
\hline Differentiation & & & & & & 0.05 \\
\hline Well differentiated & 110 & 70 & $63.6 \%$ & 40 & $36.4 \%$ & \\
\hline Poorly differentiated & 22 & 19 & $86.4 \%$ & 3 & $13.6 \%$ & \\
\hline Anaplastic & 21 & 11 & $52.4 \%$ & 10 & $47.6 \%$ & \\
\hline TPOAb & & & & & & 0.541 \\
\hline $\mathrm{N}$ & 120 & 77 & $64.2 \%$ & 43 & $35.8 \%$ & \\
\hline $\mathrm{H}$ & 27 & 19 & $70.4 \%$ & 8 & $29.6 \%$ & \\
\hline $\operatorname{Tg} A b$ & & & & & & 0.231 \\
\hline $\mathrm{N}$ & 119 & 75 & $63.0 \%$ & 44 & $37.0 \%$ & \\
\hline $\mathrm{H}$ & 28 & 21 & $75.0 \%$ & 7 & $25.0 \%$ & \\
\hline $\mathrm{T} 3$ & & & & & & 0.548 \\
\hline $\mathrm{L}$ & 6 & 5 & $83.3 \%$ & 1 & $16.7 \%$ & \\
\hline $\mathrm{N}$ & 141 & 91 & $64.5 \%$ & 50 & $35.5 \%$ & \\
\hline $\mathrm{H}$ & 2 & 1 & $50.0 \%$ & 1 & $50.0 \%$ & \\
\hline FT3 & & & & & & 0.009 \\
\hline $\mathrm{L}$ & 10 & 8 & $80.0 \%$ & 2 & $20.0 \%$ & \\
\hline $\mathrm{N}$ & 130 & 80 & $61.5 \%$ & 50 & $38.5 \%$ & \\
\hline $\mathrm{H}$ & 9 & 9 & $100.0 \%$ & 0 & $0.0 \%$ & \\
\hline $\mathrm{T} 4$ & & & & & & 0.037 \\
\hline $\mathrm{L}$ & 3 & 3 & $100.0 \%$ & 0 & $0.0 \%$ & \\
\hline $\mathrm{N}$ & 133 & 89 & $66.9 \%$ & 44 & $33.1 \%$ & \\
\hline $\mathrm{H}$ & 13 & 5 & $38.5 \%$ & 8 & $61.5 \%$ & \\
\hline FT4 & & & & & & 0.448 \\
\hline $\mathrm{L}$ & 7 & 3 & $42.9 \%$ & 4 & $57.1 \%$ & \\
\hline $\mathrm{N}$ & 135 & 89 & $65.9 \%$ & 46 & $34.1 \%$ & \\
\hline $\mathrm{H}$ & 4 & 5 & $125.0 \%$ & 2 & $50.0 \%$ & \\
\hline TSH & & & & & & 0.448 \\
\hline $\mathrm{L}$ & 7 & 6 & $85.7 \%$ & 1 & $14.3 \%$ & \\
\hline $\mathrm{N}$ & 133 & 85 & $63.9 \%$ & 48 & $36.1 \%$ & \\
\hline $\mathrm{H}$ & 9 & 6 & $66.7 \%$ & 3 & $33.3 \%$ & \\
\hline $\mathrm{Tg}$ & & & & & & 0.505 \\
\hline $\mathrm{L}$ & 14 & 11 & $78.6 \%$ & 3 & $21.4 \%$ & \\
\hline $\mathrm{N}$ & 97 & 61 & $62.9 \%$ & 36 & $37.1 \%$ & \\
\hline $\mathrm{H}$ & 36 & 24 & $66.7 \%$ & 12 & $33.3 \%$ & \\
\hline
\end{tabular}




\section{Discussion}

ATM and $\gamma \mathrm{H} 2 \mathrm{AX}$ are indicators of DSB and DDR. They are needed in the starting stage leading to DSB and could be early molecular events in carcinogenesis. H2AX is one of the substrates of ATM and could be phosphorylated by ATM. While autoactivated ATM could induce increased expression of $\gamma \mathrm{H} 2 \mathrm{AX}, \gamma \mathrm{H} 2 \mathrm{AX}$ could in turn enhance the activity of ATM [29].

Abnormal expression of ATM and $\gamma \mathrm{H} 2 \mathrm{AX}$ has been detected in various cancers with different roles. Some researches found that they were correlated with the increased risk of cancer [10-12, 19-22]. In this study, $\gamma \mathrm{H} 2 \mathrm{AX}$ and ATM showed increased expression in thyroid cancer tissues as compared with benign tissues. And $\gamma \mathrm{H} 2 \mathrm{AX}$ was correlated with ATM, which is in accordance with the previous study [29]. Both $\gamma \mathrm{H} 2 \mathrm{AX}$ and ATM play a role in thyroid cancer.

$\gamma \mathrm{H} 2 \mathrm{AX}$ was found to correlate with differentiation of thyroid cancer in this study. Well differentiated thyroid cancer tissues showed higher $\gamma \mathrm{H} 2 \mathrm{AX}$ expression level than poorly differentiated thyroid cancer tissues, while the latter showed higher $\gamma \mathrm{H} 2 \mathrm{AX}$ expression level than anaplastic thyroid cancer tissues. Statistically significant differences were also found in ATM expression among different pathologic types of thyroid cancer. Anaplastic thyroid cancer tissues showed higher ATM expression than well differentiated thyroid cancer tissues, while the latter showed higher ATM expression than poorly differentiated thyroid cancer tissues. Previous researches demonstrated that DSB-initiated genomic deletion, including ATM, was greatly increased in high-grade breast cancer [30]. Moreover, it has been proved that defects in ATM lead to poor differentiation of breast cancer [31]. Overexpression of $\gamma \mathrm{H} 2 \mathrm{AX}$ has been proved to associate with differentiation and TNM stage in gastric cancer and could serve as a potential biomarker for gastric cancer [32]. In this study, $\gamma \mathrm{H} 2 \mathrm{AX}$ and ATM expressed higher in well differentiated thyroid cancer tissues as compared to poorly differentiated thyroid cancer, which was consistent with the researches above. However, why anaplastic thyroid cancer tissue showed higher ATM expression than poorly differentiated thyroid cancer remains to be a problem. It is possibly because the specimens included in the study were insufficient. It is inappropriate to come up with a distinct correlation between ATM expression and differentiation of thyroid cancer. Anyway, more researches are needed to explore the potential relationship. Thus we proposed that $\gamma \mathrm{H} 2 \mathrm{AX}$ was correlated with differentiation of thyroid cancer while ATM was not correlated with differentiation of thyroid cancer distinctly. $\gamma \mathrm{H} 2 \mathrm{AX}$ was also found to be correlated with T classification and TNM stage. $\gamma \mathrm{H} 2 \mathrm{AX}$ was increased in patients with earlier $\mathrm{T}$ stage. A previous study showed that $\gamma \mathrm{H} 2 \mathrm{AX}$ was increased in premalignant lesions and $\gamma \mathrm{H} 2 \mathrm{AX}$ might play a critical role in the early stage of cancer [33], which is in accordance with this study. We suggested that $\gamma \mathrm{H} 2 \mathrm{AX}$ might play a more important role in early stage of thyroid cancer.

Thyroid hormones play a pivotal role in the metabolic activities and affect various organ systems. Zambrano et al. have proved that T3 could activate ATM-dependent adenosine monophosphate-activated protein kinase (PRKAA) signaling by combining with THRB (thyroid hormone receptor beta), thus playing a pivotal role in mitochondrial respiration and leading to DSB in mouse embryonic fibroblasts [34]. In the absence of ATM, T3 could not induce senescence, whereas transfection of ATM restored the response to T3 [34]. Associations between thyroid functions tests and ATM and $\gamma \mathrm{H} 2 \mathrm{AX}$ expression were also analyzed in this study. We found no correlation between T3 and the expression of ATM and $\gamma \mathrm{H} 2 \mathrm{AX}$. However, the results showed that both of ATM and $\gamma \mathrm{H} 2 \mathrm{AX}$ were correlated with FT3 in thyroid cancer. FT3 could be recognized as a marker of euthyroid syndrome in various diseases, and reduced FT3 in plasma could diminish the conversion of T4 to T3 [35]. Recent researches indicated that lower FT3 and FT4 levels and higher TSH level were correlated with thyroid cancer risk [28]. $\gamma \mathrm{H} 2 \mathrm{AX}$ was also associated with FT4 and TSH in this study. Although the results are inconclusive, we speculated an association between thyroid disorders and ATM and $\gamma \mathrm{H} 2 \mathrm{AX}$ expression.

Overall, both $\gamma \mathrm{H} 2 \mathrm{AX}$ and ATM were supposed to influence thyroid hormones and $\gamma \mathrm{H} 2 \mathrm{AX}$ has been proved to play a role in the differentiation status of thyroid cancer. Since ATM activation and $\gamma \mathrm{H} 2 \mathrm{AX}$ have been recognized as indicators of DNA damage, more researches are needed for further discussing the relationship between thyroid functions and DNA damage.

\section{Conflict of Interests}

All the authors declare that there is no conflict of interests regarding the publication of this paper.

\section{Authors' Contribution}

Jin-lin $\mathrm{Hu}, \mathrm{Si}$-si Hu, Xiu-xiu Hou, and Xin Zhu contributed equally to this study.

\section{Acknowledgments}

This study was supported by Ministry of Health P. R. China Foundation for Science Research (WKJ2012-2-021) and Zhejiang Provincial Program for the Cultivation of HighLevel Innovative Health Talents (Health Bureau of Zhejiang Province extended 2008-134).

\section{References}

[1] R. Siegel, D. Naishadham, and A. Jemal, "Cancer statistics, 2012," CA: A Cancer Journal for Clinicians, vol. 62, no. 1, pp. 10-29, 2012.

[2] L. Davies and H. G. Welch, "Increasing incidence of thyroid cancer in the United States, 1973-2002," The Journal of the American Medical Association, vol. 295, no. 18, pp. 2164-2167, 2006.

[3] R. C. Smallridge, L. A. Marlow, and J. A. Copland, "Anaplastic thyroid cancer: molecular pathogenesis and emerging therapies," Endocrine-Related Cancer, vol. 16, no. 1, pp. 17-44, 2009. 
[4] S. P. Jackson, "Sensing and repairing DNA double-strand breaks," Carcinogenesis, vol. 23, no. 5, pp. 687-696, 2002.

[5] M. Podhorecka, A. Skladanowski, and P. Bozko, "H2AX phosphorylation: its role in DNA damage response and cancer therapy," Journal of Nucleic Acids, vol. 2010, Article ID 920161, 9 pages, 2010.

[6] O. Fernandez-Capetillo, A. Lee, M. Nussenzweig, and A. Nussenzweig, "H2AX: the histone guardian of the genome," DNA Repair, vol. 3, no. 8-9, pp. 959-967, 2004.

[7] K. Savitsky, A. Bar-Shira, S. Gilad et al., "A single ataxia telangiectasia gene with a product similar to PI-3 kinase," Science, vol. 268, no. 5218, pp. 1749-1753, 1995.

[8] F. A. Derheimer and M. B. Kastan, "Multiple roles of ATM in monitoring and maintaining DNA integrity," FEBS Letters, vol. 584, no. 17, pp. 3675-3681, 2010.

[9] L. Xu, E. C. Morari, Q. Wei, E. M. Sturgis, and L. S. Ward, "Functional variations in the ATM gene and susceptibility to differentiated thyroid carcinoma," Journal of Clinical Endocrinology and Metabolism, vol. 97, no. 6, pp. 1913-1921, 2012.

[10] Y.-L. Zheng, O. Kosti, C. A. Loffredo et al., "Elevated lung cancer risk is associated with deficiencies in cell cycle checkpoints: genotype and phenotype analyses from a case-control study," International Journal of Cancer, vol. 126, no. 9, pp. 2199-2210, 2010.

[11] T. A. Buchholz, M. M. Weil, C. L. Ashorn et al., "A Ser49Cys variant in the ataxia telangiectasia, mutated, gene that is more common in patients with breast carcinoma compared with population controls," Cancer, vol.100, no. 7, pp. 1345-1351, 2004.

[12] S. Angèle, A. Falconer, S. M. Edwards et al., "ATM polymorphisms as risk factors for prostate cancer development," British Journal of Cancer, vol. 91, no. 4, pp. 783-787, 2004.

[13] J. H. Barrett, M. M. Iles, and M. Harland, "Genome-wide association study identifies three new melanoma susceptibility loci," Nature Genetics, vol. 43, no. 11, pp. 1108-1113, 2011.

[14] A. Wójcicka, M. Czetwertyńska, M. Świerniak et al., "Variants in the ATM-CHEK2-BRCA1 axis determine genetic predisposition and clinical presentation of papillary thyroid carcinoma," Genes Chromosomes and Cancer, vol. 53, no. 6, pp. 516-523, 2014.

[15] J. Kang, X.-Z. Deng, Y.-B. Fan, and B. Wu, "Relationships of FOXE1 and ATM genetic polymorphisms with papillary thyroid carcinoma risk: a meta-analysis," Tumor Biology, vol. 35, no. 7, pp. 7085-7096, 2014.

[16] C. M. Song, T.-K. Kwon, B. L. Park, Y. B. Ji, and K. Tae, "Single nucleotide polymorphisms of ataxia telangiectasia mutated and the risk of papillary thyroid carcinoma," Environmental and Molecular Mutagenesis, vol. 56, no. 1, pp. 70-76, 2015.

[17] Y. Gu, Y. Yu, L. Ai et al., "Association of the ATM gene polymorphisms with papillary thyroid cancer," Endocrine, vol. 45, no. 3, pp. 454-461, 2014.

[18] F. Damiola, G. Byrnes, M. Moissonnier et al., "Contribution of ATM and FOXE1 (TTF2) to risk of papillary thyroid carcinoma in Belarusian children exposed to radiation," International Journal of Cancer, vol. 134, no. 7, pp. 1659-1668, 2014.

[19] M. B. Kastan and D.-S. Lim, "The many substrates and functions of ATM," Nature Reviews Molecular Cell Biology, vol. 1, no. 3, pp. 179-186, 2000.

[20] K. Oka, T. Tanaka, T. Enoki et al., "DNA damage signaling is activated during cancer progression in human colorectal carcinoma," Cancer Biology and Therapy, vol. 9, no. 3, pp. 246252, 2010 .
[21] D. Matthaios, P. Hountis, P. Karakitsos, D. Bouros, and S. Kakolyris, "H2AX a promising biomarker for lung cancer: a review," Cancer Investigation, vol. 31, no. 9, pp. 582-599, 2013.

[22] Y. Matsuda, T. Wakai, M. Kubota et al., "DNA damage sensor $\gamma-\mathrm{H} 2 \mathrm{AX}$ is increased in preneoplastic lesions of hepatocellular carcinoma," The Scientific World Journal, vol. 2013, Article ID 597095, 7 pages, 2013.

[23] G. Z. Sisi Hu, J. Xu, X. Zhu et al., "Disparity expression of gammaH2AX in papillary thyroid cancer and nodular goiter," Clinical Laboratory, vol. 60, no. 12, pp. 2031-2037, 2014.

[24] J. Galleani, C. Miranda, M. A. Pierotti, and A. Greco, "H2AX phosphorylation and kinetics of radiation-induced DNA double strand break repair in human primary thyrocytes," Thyroid, vol. 19, no. 3, pp. 257-264, 2009.

[25] M. Lassmann, H. Hänscheid, D. Gassen et al., "In vivo formation of $\gamma$-H2AX and 53BP1 DNA repair foci in blood cells after radioiodine therapy of differentiated thyroid cancer," The Journal of Nuclear Medicine, vol. 51, no. 8, pp. 1318-1325, 2010.

[26] M. Doai, N. Watanabe, T. Takahashi et al., "Sensitive immunodetection of radiotoxicity after iodine-131 therapy for thyroid cancer using gamma-H2AX foci of DNA damage in lymphocytes," Annals of Nuclear Medicine, vol. 27, no. 3, pp. 233-238, 2013.

[27] C. E. Caldon, "Estrogen signaling and the DNA damage response in hormone dependent breast cancers," Frontiers in Oncology, vol. 4, article 106, 2014.

[28] K. Gul, D. Ozdemir, A. Dirikoc et al., "Are endogenously lower serum thyroid hormones new predictors for thyroid malignancy in addition to higher serum thyrotropin?" Endocrine, vol. 37, no. 2, pp. 253-260, 2010.

[29] S. Bandi, P. Viswanathan, and S. Gupta, "Evaluation of cytotoxicity and DNA damage response with analysis of intracellular ATM signaling pathways," Assay and Drug Development Technologies, vol. 12, no. 5, pp. 272-281, 2014.

[30] C.-Y. Shen, J.-C. Yu, Y.-L. Lo et al., "Genome-wide search for loss of heterozygosity using laser capture microdissected tissue of breast carcinoma: an implication for mutator phenotype and breast cancer pathogenesis," Cancer Research, vol. 60, no. 14, pp. 3884-3892, 2000.

[31] S. L. Ding, L. F. Sheu, J. C. Yu et al., "Abnormality of the DNA double-strand-break checkpoint/repair genes, ATM, BRCA1 and TP53, in breast cancer is related to tumour grade," British Journal of Cancer, vol. 90, no. 10, pp. 1995-2001, 2004.

[32] C. Xie, L. Y. Xu, Z. Yang et al., "Expression of gammaH2AX in various gastric pathologies and its association with Helicobacter pylori infection," Oncology Letters, vol. 7, no. 1, pp. 159-163, 2014.

[33] L.-J. Mah, A. El-Osta, and T. C. Karagiannis, " $\gamma \mathrm{H} 2 \mathrm{AX}$ as a molecular marker of aging and disease," Epigenetics, vol. 5, no. 2, pp. 129-136, 2010.

[34] A. Zambrano, V. García-Carpizo, M. E. Gallardo et al., "The thyroid hormone receptor $\beta$ induces DNA damage and premature senescence," Journal of Cell Biology, vol. 204, no. 1, pp. 129-146, 2014.

[35] E. M. Abdel-Rahman, W. Mansour, and J. L. Holley, "Thyroid hormone abnormalities and frailty in elderly patients with chronic kidney disease: a hypothesis," Seminars in Dialysis, vol. 23, no. 3, pp. 317-323, 2010. 


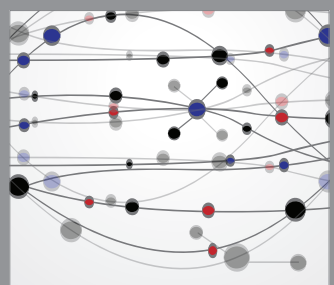

The Scientific World Journal
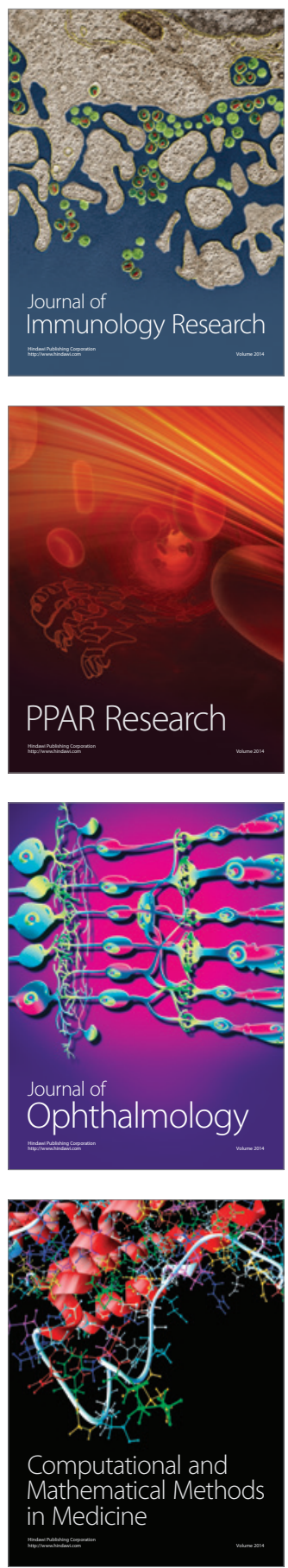

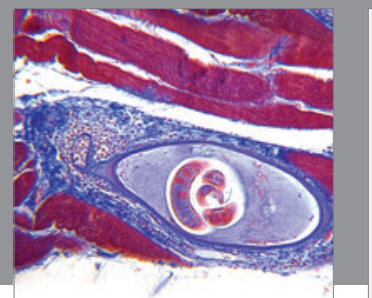

Gastroenterology

Research and Practice
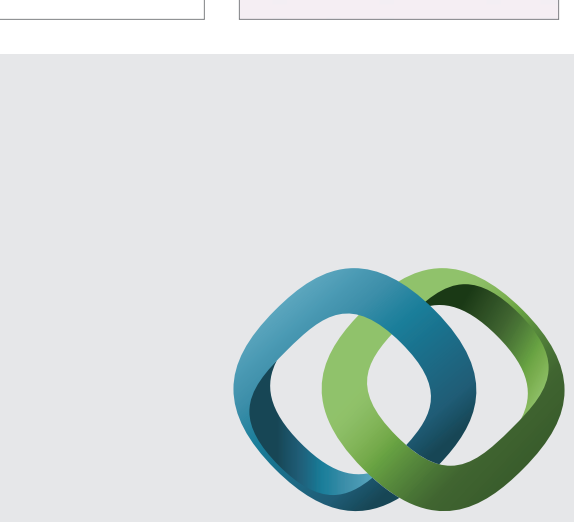

\section{Hindawi}

Submit your manuscripts at

http://www.hindawi.com
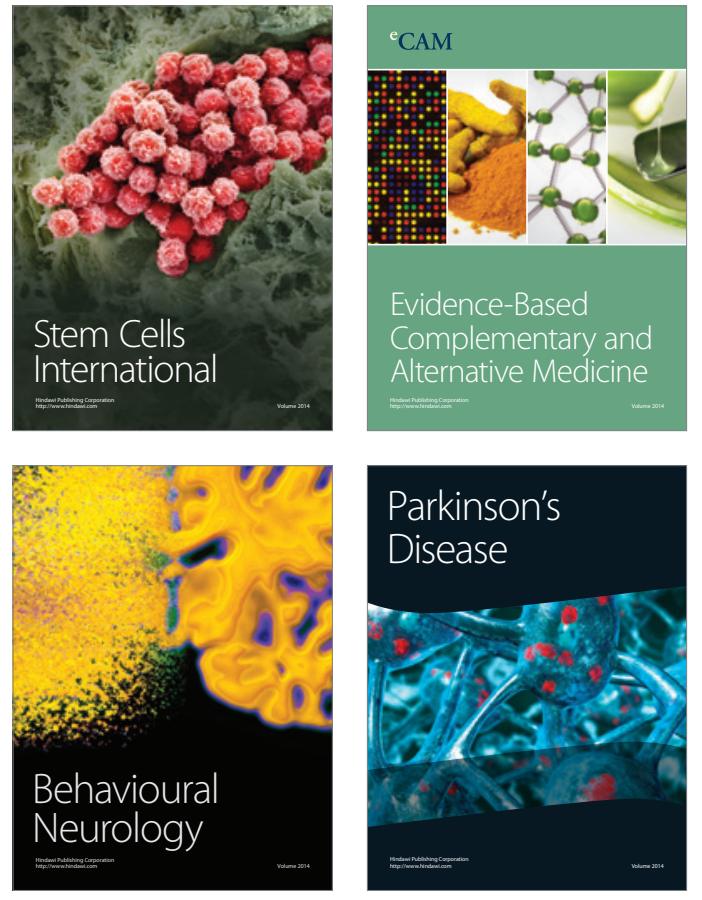
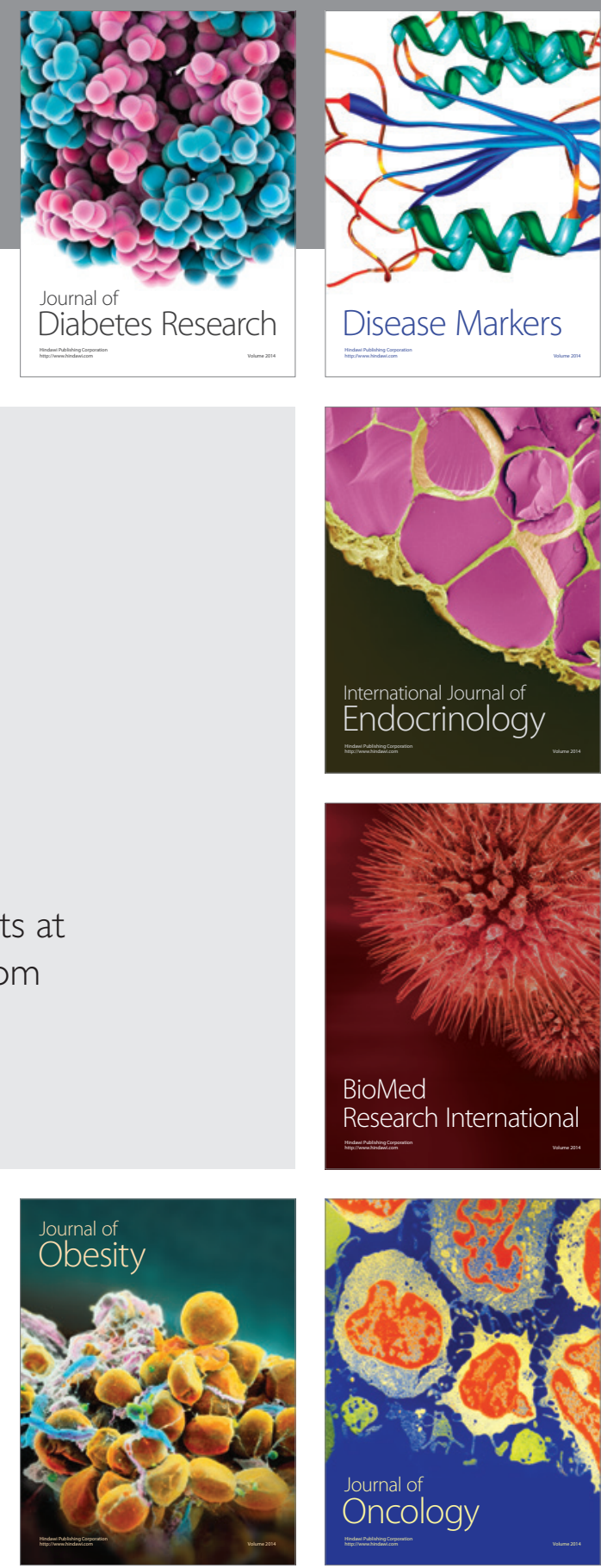

Disease Markers
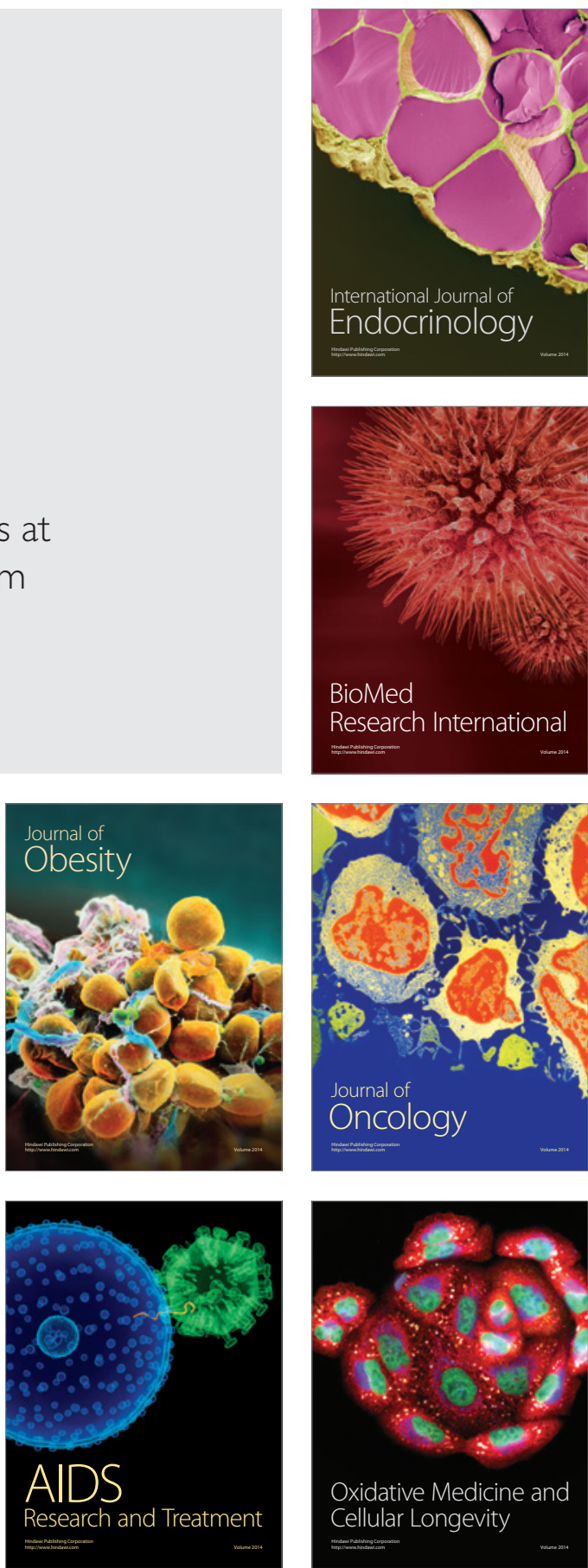